\title{
Reduce Order Modelling of Power System Models Using Interpolatory Projections Technique
}

\author{
Mohammad-Sahadet Hossain and M. Monir Uddin
}

\begin{abstract}
Large, complex dynamical systems, such as, power systems, are very challenging task to model and analysis. Numerous techniques have been developed to handle the difficulties arising from the size and complexity of typical realistic power system models. These complexities demand to formulate reduced order dynamic equivalent models of power systems in many applications and studies. Linearizing around the equilibrium point, a stable time invariant power system model leads to index 1 differential-algebraic (DAE) system. A balancing based model reduction technique for such a system is discussed in a paper of $F$. Freitas et al. in 2008. The main drawback of this method is to compute two Gramian factors of the system by solving two continuous-time algebraic Lyapunov equations. On the other hand interpolatory model reduction via iterative rational Krylov algorithm (IRKA) is computationally efficient since it requires only matrix-vector products or linear solvers. This paper contributes an interpolatory technique using IRKA for a class of index 1 DAE systems to obtain reduced standard ordinary differential (ODE) systems. We also show that a simple algebraic manipulation retrieve reduced index-1 DAE systems. The proposed technique is applied to a data of linearized power system models. Numerical results illustrate the efficiency of the techniques.
\end{abstract}

Index Terms-Descriptor systems, indices of descriptor systems, interpolatory projection, model reduction, power systems, rational Krylov approximation.

\section{INTRODUCTION}

Formulate reduced-order dynamic equivalent models of power systems are very desirable, specially in the sense of fast and cost-efficient stability assessment. By linearizing a stable time invariant power system model around the equilibrium point one may come up with descriptor systems of index 1. See, e.g.,[1]-[5] for details. The general framework for these descriptor systems is to formulate an equivalent ODE system of the corresponding power system model associated with differential-algebraic equations (DAEs). We discuss interpolatory projection based model reduction of descriptor system, or DAEs, given by

$$
\underbrace{\left[\begin{array}{cc}
E_{1} & 0 \\
0 & 0
\end{array}\right]}_{\tilde{E}}\left[\begin{array}{l}
\dot{x}_{1}(t) \\
\dot{x}_{2}(t)
\end{array}\right]=\underbrace{\left[\begin{array}{cc}
A_{1} & A_{2} \\
A_{3} & A_{4}
\end{array}\right]}_{\widetilde{A}}\left[\begin{array}{l}
x_{1}(t) \\
x_{2}(t)
\end{array}\right]+\left[\begin{array}{c}
B_{1}(t) \\
B_{2}(t)
\end{array}\right] u(t),
$$

Manuscript received March 29, 2015; revised May 19, 2015. This work was supported in part by the Travel and Research Grant of North South University, Dhaka.

Mohammad-Sahadet Hossain was with Max Planck Institute for Dynamics of Complex Technical Systems, Magdeburg, Germany. He is now with the Department of Mathematics and Physics, North South University, Dhaka, Bangladesh (e-mail: mohammad.hossain@northsouth.edu).

M. Monir Uddin is with the Max Planck Institute for Dynamics of Complex Technical Systems, Sandtorstr. 1, D-39106 Magdeburg, Germany (e-mail: uddin@mpi-magdeburg.mpg.de).

$$
\hat{y}(t)=\underbrace{\left[\begin{array}{ll}
C_{1} & C_{2}
\end{array}\right]}_{\tilde{c}}\left[\begin{array}{l}
x_{1}(t) \\
x_{2}(t)
\end{array}\right]+D u(t)
$$

where $x_{1}(t) \in \mathbb{R}^{n_{1}}, x_{2}(t) \in \mathbb{R}^{n_{2}}$ are the states, $u(t) \in \mathbb{R}^{p}$ is the control input to the system and the measurement output is $y(t) \in \mathbb{R}^{m}$. Here $\tilde{E}, \tilde{A}, \tilde{B}$, and $\tilde{C}$ are all matrices with appropriate dimensions. We assume that $\tilde{E}$ is singular and the block matrix $\mathrm{A}_{4}$ is nonsingular. Therefore, (1) is defined as index 1 descriptor system.

Since the block matrix A4 is invertible, the second line of the first equation in (1) gives

$$
x_{2}(t)=-A_{4}^{-1} A_{3} x_{1}(t)+A_{4}^{-1} B_{2} u(t) .
$$

Now inserting the $x_{2}(t)$ into the first line of the first equation (1) and in the output equation, i.e., in the second equation of (1), the descriptor system (1) leads to ODE system in the state-space form

$$
\begin{aligned}
E \dot{x}_{1}(t) & =A x_{1}(t)+B u(t), \\
y(t) & =C x_{1}(t)+D_{a} u(t),
\end{aligned}
$$

where

$$
\begin{aligned}
& E:=E_{1}, \\
& A:=A_{1}-A_{2} A_{4}^{-1} A_{3}, \quad B:=B_{1}-A_{2} A_{4}^{-1} B_{2}, \\
& C:=C_{1}-C_{2} A_{4}^{-1} A_{3}, \quad D_{a}:=D-C_{2} A_{4}^{-1} B_{2} .
\end{aligned}
$$

Dynamical systems (1) and (2) are equivalent, since both have the same finite spectrum and they are different realizations of the same transfer function. Transfer function (or transfer function matrix) is the input-output relation of the system in the frequency domain which can be defined by

$$
G(s)=\bar{C}(s \bar{E}-\bar{A})^{-1} \bar{B}+\bar{D}
$$

where $s \in \mathbb{C}$, and and $(\bar{E}, \bar{A}, \bar{B}, \bar{C}, \bar{D})$ in $\mathrm{G}(\mathrm{s})$ is either $(\tilde{E}, \tilde{A}, \tilde{B}, \tilde{C}, D)$ or $\quad\left(E, A, B, C, D_{a}\right)$. Although, all the block matrices in (1) are sparse, computing the A, B and C matrices explicitly inverting $\mathrm{A}_{4}$ makes the system (2) dense [6] which might lead to computational complexity. Therefore, explicit formulation of $\mathrm{A}, \mathrm{B}$ and $\mathrm{C}$ is avoided in practice. By reduce order modeling (ROM) we mean to replace (2) by $r$ ( $r$ $\left.<<n_{1}\right)$, a much lower dimensional surrogate system

$$
\begin{aligned}
\hat{E} \dot{\hat{x}}(t) & =\hat{A} \hat{x}(t)+\hat{B} u(t), \\
\hat{y}(t) & =\hat{C} \hat{x}(t)+\hat{D}_{a} u(t),
\end{aligned}
$$


where $\hat{E}, \hat{A} \in \mathbb{R}^{r \times r}, \hat{B} \in \mathbb{R}^{r \times p}, \hat{C} \in \mathbb{R}^{m \times r}$, and $\hat{D}_{a} \in D_{a}$. The reduce order model here is supposed to fulfill some certain approximation requirements, for instance the approximation error $\|y(t)-\hat{y}(t)\|$, or correspondingly $\|G()-.\hat{G}()$.$\| , where$ $\hat{G}($.) is the transfer function matrix of the reduce order model, should be small in some suitable norms, e.g., the $\mathbf{H}_{\infty}$ or $\mathbf{H}_{2}$ norms (see [7]). The way how to achieve this goal is called model order reduction (MOR). See, e.g., [7]-[10] for motivations, applications, restrictions and techniques of MOR.

Among several model reduction techniques, the balanced truncation (BT) and the interpolatory technique via iterative Krylov algorithm (IRKA) are the two prominent methods for large-scale dynamical systems. Although balanced truncation has a priori error bound and it guarantees the system stability, the implementation of this this prominent method is expensive since it requires to solve two continuous-time algebraic Lyapunov equations. On the other hand implementation of interpolatory projection via IRKA is simple because it requires only matrix-vector products or linear solvers. A balanced truncation method for the index 1 DAE system (1) has already been discussed in [5]. Here we contribute the interpolatory method via IRKA.

The concept of projection for interpolatory model reduction was initially introduced in [11] and later Grimme in [12] modified the approach by utilizing the rational Krylov method [13]. Since Krylov based methods can achieve moment matching without explicitly computing moments (explicit computation of moments is known to be ill-conditioned [14]), they are extremely useful for model reduction of large scale systems. The quality of the reduced model is highly dependent on the choice of interpolation points and therefore various techniques [11] have been developed for the selection of interpolation points.

Recently in [15], the issue of selecting a good set of interpolation points is linked to the problem of $\mathbf{H}_{2}$-optimal model reduction. The iterative rational Krylov algorithm (IRKA) is proposed in [15] which identify a good choice of interpolation points that guarantees the $\mathbf{H}_{2}$-optimality conditions for the reduce system. Starting from an initial set of interpolation points, the IRKA iterations update the interpolation points until they converge to some fix values. Until now we have considered that (1) is a single-input single-output (SISO) system. A complete procedure of IRKA for a SISO system is given in [15, Algorithm 4.1]. For model reduction of multi-input multi-output (MIMO) dynamical systems, rational tangential interpolation has been developed by Gallivan et al. [16]. In this paper we emphasize on the case of MIMO system.

Very recently model reduction of rational tangential interpolation via IRKA is extended for the descriptor system in [17]. In principle there one has to compute the spectral projectors onto the left and right deflating subspaces corresponding to the finite eigenvalues of the system. Although the projectors are available for models of particular structure (including the system (1), computation is expensive. However, the authors in [17] show that for a particular structured index 1 DAEs, spectral projectors are not required in the implementation of MOR. In this paper we also avoid the computation of the spectral projectors. In contrast to [17], our index 1 DAEs is slightly different and also in the implementation techniques

The main contribution of this paper is to form (2) implicitly and work on the sparse formulation of the original model (1). Moreover, a small algebraic manipulation of (4) turns out a reduced second order index- 1 model if that is desired which is not possible in the case of [17].

\section{PReliminaries}

To precise the MOR technique for the DAEs (1) via interpolatory methods, in this section we discuss the method for the generalized linear time-invariant (LTI) continuoustime system

$$
\begin{aligned}
\mathcal{E} \dot{x}(t) & =\mathcal{A} x(t)+\mathcal{B} u(t), \\
y(t) & =C x(t)+\mathcal{D} u(t),
\end{aligned}
$$

In which $\mathcal{E} \in \mathbb{R}^{n \times n}$ is non-singular, and $\mathcal{A} \in \mathbb{R}^{n \times n}$, $\mathcal{B} \in \mathbb{R}^{n \times \mathrm{p}}, C \in \mathbb{R}^{m \times n}$ and $\mathcal{D} \in \mathbb{R}^{m \times \mathrm{p}}$. We want to construct a $r(r<<\mathrm{n})$ dimensional reduce system

$$
\begin{aligned}
& \hat{E} \dot{\hat{x}}(t)=\hat{A} \hat{x}(t)+\hat{B} u(t), \\
& \hat{y}(t)=\hat{C} \hat{x}(t)+\hat{D} u(t),
\end{aligned}
$$

where

$$
\begin{gathered}
\hat{\mathcal{E}}=\mathcal{T}_{\ell}^{\mathrm{T}} \mathcal{E} \mathcal{T}_{r}, \hat{\mathcal{A}}=\mathcal{T}_{\ell}^{\mathrm{T}} \mathcal{A} \mathcal{T}_{r}, \\
\hat{\mathcal{B}}=\mathcal{T}_{\ell}^{\mathrm{T}} \mathcal{B}, \hat{C}=C \mathcal{T}_{r}, \hat{\mathcal{D}}:=\mathcal{D} .
\end{gathered}
$$

Here $\mathcal{T}_{\ell} \in \in \mathbb{R}^{n \times \mathrm{r}} \quad$ and $\quad \mathcal{T}_{r} \in \in \mathbb{R}^{n \times \mathrm{r}} \quad$ are called, respectively, the left and the right transformation matrices.

For the time being, we consider the case where (5) is a single input single output (SISO) system (i.e., $\mathcal{B} \in \mathbb{R}^{n \times 1}$ and $C \in \mathbb{R}^{1 \times n}$ ). Interpolatory projection methods seek a ROM (6) by constructing the matrices $\mathcal{T}_{\ell}$ and $\mathcal{T}_{r}$ in such way that the reduced transfer function interpolates the original transfer function at a predefined set of interpolation points. That is to find $\hat{G}\left(\alpha_{i}\right)$ such that, for $i=1,2, \ldots ., r$,

$$
G\left(\alpha_{i}\right)=\hat{G}\left(\alpha_{i}\right),
$$

where $\alpha_{i} \in \mathbb{C}$ are the interpolation points. Often, in addition to the above conditions, we are interested in matching more quantities, that is

$$
G^{(j)}\left(\alpha_{i}\right)=\hat{G}^{(j)}\left(\alpha_{i}\right), \text { for } j=0,1, \ldots, q,
$$

where $C\left(\alpha_{i} \mathcal{E}-\mathcal{A}\right)^{-(j+1)} \mathcal{B}$ is called the $j$-th moment of $\mathrm{G}(\mathrm{s})$ at $\alpha_{i}$ and represents the $j$-th derivative of $\mathrm{G}(\mathrm{s})$ evaluated at $\sigma_{i}$. Note that for $j=0$, these conditions reduce to (8). In 
this paper, we restrict ourself to simple Hermite interpolation, where $\mathrm{j}=0$ and $\mathrm{j}=1$. In the following, we discuss how projection can ensure reduced interpolating approximation, by carefully selecting the matrices $\mathcal{T}_{\ell}$ and $\mathcal{T}_{r}$.

The following results suggest a choice of $\mathcal{T}_{\ell}$ and $\mathcal{T}_{r}$ that ensures Hermite interpolation with the use of rational Krylov subspace.

Lemma 2.1 ([15]): Consider two sets of distinct interpolation points, $\left\{\alpha_{i}\right\}_{i=1}^{r} \in \mathbb{C}$ and $\left\{\beta_{i}\right\}_{i=1}^{r} \in \mathbb{C}$, which are closed under conjugation (i.e., the points are either real or appear in conjugate pairs). Suppose $\mathcal{T}_{\ell}$ and $\mathcal{T}_{r}$ satisfy the following

$$
\operatorname{Range}\left\{\mathcal{T}_{r}\right\}=\operatorname{span}\left\{\left(\alpha_{1} \mathcal{E}-\mathcal{A}\right)^{-1} \mathcal{B}, \ldots, \quad\left(\alpha_{r} \mathcal{E}-\mathcal{A}\right)^{-1} \mathcal{B}\right\}
$$

$\operatorname{Range}\left\{\mathcal{T}_{\ell}\right\}=\operatorname{span}\left\{\left(\beta_{1} \mathcal{E}-\mathcal{A}\right)^{-\mathrm{T}} C^{\mathrm{T}}, \cdots, \quad\left(\beta_{r} \mathcal{E}-\mathcal{A}\right)^{-\mathrm{T}} C^{\mathrm{T}}\right\}$

Then $\mathcal{T}_{\ell}$ and $\mathcal{T}_{r} \quad$ can be chosen real and $\hat{G}(s)=\hat{C}(s \hat{E}-\hat{A})^{-1} \hat{B}$ where $\hat{E}, \hat{A}, \hat{B}, \hat{C}$ are as defined in (6), satisfies the Hermite interpolation conditions

$$
G\left(\alpha_{i}\right)=\hat{G}\left(\alpha_{i}\right), \quad G\left(\beta_{i}\right)=\hat{G}\left(\beta_{i}\right),
$$

and

$$
G^{\prime}\left(\alpha_{i}\right)=\hat{G}^{\prime}\left(\alpha_{i}\right) \text {, when } \alpha_{i}=\beta_{i} \text {, for } i=1,2, \ldots, \mathrm{r} \text {, }
$$

where $G^{\prime}\left(\alpha_{i}\right)$ and $\hat{G}^{\prime}\left(\alpha_{i}\right)$ are, respectively, the first derivatives of $G\left(\alpha_{i}\right)$ and $\hat{G}\left(\alpha_{i}\right)$.

The subspace in (10), that is the span of the column vectors $\left(\alpha_{i} \mathcal{E}-\mathcal{A}\right)^{-1} \mathcal{B}$, for $i=1,2, \ldots ., r$, can be considered as the union of shifted rational Krylov subspaces. For a given shift frequency $\alpha \in \mathbb{C}$, the rational Krylov subspace $\kappa_{q}\left((\alpha \mathcal{E}-\mathcal{A})^{-1}, \quad(\alpha \mathcal{E}-\mathcal{A})^{-1} \mathcal{B}\right)$ is defined as

$$
\begin{aligned}
& \kappa_{q}\left((\alpha \mathcal{E}-\mathcal{A})^{-1}, \quad(\alpha \mathcal{E}-\mathcal{A})^{-1} \mathcal{B}\right):= \\
& \operatorname{span}\left\{(\alpha \mathcal{E}-\mathcal{A})^{-1} \mathcal{B}, \cdots,(\alpha \mathcal{E}-\mathcal{A})^{-q} \mathcal{B}\right\} .
\end{aligned}
$$

If $q=1$ for each $\alpha_{i}, i=1,2, \ldots ., r$, then the union of such shifted rational Krylov subspaces is equivalent to the subspace in (10). Analogously, the subspace in (11) can be also be defined as the union of shifted rational Krylov subspaces given above. Thus to summarize, rational Krylov based model reduction requires a suitable choice of interpolation points, the construction of $\mathcal{T}_{\ell}$ and $\mathcal{T}_{r}$ as in Lemma 2.1 and the use of Petrov-Galerkin conditions [18].

The quality of the reduced model is highly dependent on the choice of interpolation points and therefore various techniques [11] have been developed for the selection of interpolation points. Recently in [15], the issue of selecting a good choice of interpolation points is linked to the problem of
$\mathbf{H}_{2}$-optimal model reduction.

Definition 2.1: A ROM (6) is called $\mathbf{H}_{2}$-optimal if it satisfies

$$
\|G\|_{\mathbf{H}_{2}}=\min _{\operatorname{dim}(\hat{G})=r}\|G-\hat{G}\|_{\mathbf{H}_{2}}
$$

For SISO systems, IRKA is proposed in [15] which prominently finds an efficient choice of interpolation points that guarantees the $\mathbf{H}_{2}$-optimality for the reduced system. The procedure starts with an initial set of interpolation points, and it updates the interpolation points until the iterations converge to a reference value.

Model reduction of MIMO dynamical systems, rational tangential interpolation has been developed by Gallivan et.al. [16]. The problem of rational tangential interpolation is to construct $\mathcal{T}_{\ell}$ and $\mathcal{T}_{r}$ such that the reduced transfer function $\hat{G}(s)$ tangentially interpolate the original transfer function $\mathrm{G}(\mathrm{s})$ at a predefined set of interpolation points and some fixed tangent directions. That is

$$
\mathrm{G} \alpha_{i} b_{i}=\hat{G} \alpha_{i} b_{i}, \quad c_{i}^{T} \mathrm{G} \alpha_{i}=c_{i}^{T} \hat{G} \alpha_{i},
$$

and

$$
c_{i}^{T} \mathrm{G} \alpha_{i} b_{i}=c_{i}^{T} \hat{G} \alpha_{i} b_{i}, \text { for } i=1,2, \ldots, \mathrm{r},
$$

where $b_{i} \in \mathbb{C}^{m}$ and $c_{i} \in \mathbb{C}^{p}$ are the right and left tangential directions, respectively, and correspond to the interpolation points $\alpha_{i}$. With these quantities, the rational tangential interpolation can be achieved. The IRKA based interpolatory projection methods for the MIMO systems have been discussed in [15], where the algorithm updates interpolation points as well as tangential directions until the reduced system satisfy interpolate based necessary condition for $\mathbf{H}_{2}$ -optimality. We have summarized a complete procedure of such method for MIMO system in Algorithm 1.

Algorithm 1: IRKA for MIMO systems in (5)

Input: $\mathcal{E}, \mathcal{A}, \mathcal{B}, C$ and $\mathcal{D}$ from (5).

Output: Reduce dimensional matrices $\widehat{\varepsilon}, \hat{E}, \hat{A}, \hat{B}, \hat{C}$, and $\hat{\mathcal{D}}$ as in (6).

1. Make an initial selection of the interpolation points $\left\{\alpha_{i}\right\}_{i=1}^{r} \in \mathbb{C}$, and the tangential directions and $b_{i}{ }_{i=1}^{r}$, and $c_{i}{ }_{i=1}^{r}$.

\section{Construct}

$$
\begin{aligned}
& \mathcal{T}_{r}=\left[\left(\alpha_{1} \mathcal{E}-\mathcal{A}\right)^{-1} \mathcal{B} \mathrm{b}_{1}, \cdots, \quad\left(\alpha_{r} \mathcal{E}-\mathcal{A}\right)^{-1} \mathcal{B} \mathrm{b}_{r}\right], \\
& \mathcal{T}_{\ell}=\left[\left(\alpha_{1} \mathcal{E}-\mathcal{A}\right)^{-\mathrm{T}} C^{\mathrm{T}} \mathrm{c}_{1}, \cdots, \quad\left(\alpha_{r} \mathcal{E}-\mathcal{A}\right)^{-\mathrm{T}} C^{\mathrm{T}} \mathrm{c}_{\mathrm{r}}\right],
\end{aligned}
$$

3. while (not converge) do

4. $\hat{\mathcal{E}}=\mathcal{T}_{\ell}^{\mathrm{T}} \mathcal{E} \mathcal{T}_{r}, \hat{\mathcal{A}}=\mathcal{T}_{\ell}^{\mathrm{T}} \mathcal{A} \mathcal{T}_{r}$, 


$$
\hat{\mathcal{B}}=\mathcal{T}_{\ell}^{\mathrm{T}} \mathcal{B}, \hat{C}=C \mathcal{T}_{r} .
$$

\section{Compute}

$$
\begin{gathered}
\hat{\mathcal{A}} z_{i}=\hat{\lambda}_{i} \hat{\mathcal{E}} z_{i} \text { and } y^{*} \hat{\mathcal{A}}=\hat{\lambda}_{i} \mathcal{Y}^{*} \hat{\mathcal{E}} z_{i} . . \\
\begin{array}{l}
\text { 6. } \alpha_{i} \leftarrow-\hat{\lambda}_{i}, \mathrm{~b}_{i}^{\mathrm{T}} \leftarrow \mathcal{Y}^{*} \hat{\mathcal{B}}, \text { and } \mathrm{c}_{i} \leftarrow \hat{\mathcal{C}} z_{i} \\
\text { for } i=1,2, \ldots, \mathrm{r} .
\end{array} \\
\text { 7. } \mathcal{T}_{r}=\left[\left(\alpha_{1} \mathcal{E}-\mathcal{A}\right)^{-1} \mathcal{B} \mathrm{b}_{1}, \cdots, \quad\left(\alpha_{r} \mathcal{E}-\mathcal{A}\right)^{-1} \mathcal{B} \mathrm{b}_{r}\right], \\
\text { 8. } \mathcal{T}_{\ell}=\left[\left(\alpha_{1} \mathcal{E}-\mathcal{A}\right)^{-\mathrm{T}} C^{\mathrm{T}} \mathrm{c}_{1}, \cdots, \quad\left(\alpha_{r} \mathcal{E}-\mathcal{A}\right)^{-\mathrm{T}} C^{\mathrm{T}} \mathrm{c}_{\mathrm{r}}\right] . \\
\text { 9. } i=i+1 \\
\text { 10. end while } \\
\text { 11. Construct the reduced matrices as in (7). }
\end{gathered}
$$

\section{Model Reduction OF INDEX 1 DAEs}

However, Algorithm 1 can be generalized for the model reduction of power systems associated with index 1 DAEs (1). For this purpose first we need to convert the DAEs (1) into the ODE system (2). The right and left transformation matrices as defined in (14) then can be formed as

$$
\begin{gathered}
T_{r}=\left[\left(\alpha_{1} E-A\right)^{-1} B b_{1}, \cdots, \quad\left(\alpha_{r} E-A\right)^{-1} B b_{r}\right], \\
T_{l}=\left[\left(\alpha_{1} E-A\right)^{-T} C^{T} c_{1}, \cdots, \quad\left(\alpha_{r} E-A\right)^{-T} C^{T} c_{r}\right] .
\end{gathered}
$$

A close observation reveals that to generate each term of $T_{r}$, we have to solve a shifted linear system like

$$
(\alpha E-A) v=B b
$$

which is equivalent to

$$
\alpha E_{1}-A_{1}-A_{2} A_{4}^{-1} A_{3} \quad v=\left(B_{1}-A_{2} A_{4}^{-1} B_{2}\right) b .
$$

Recalling the strategy in [5] instead of solving the above linear system we can solve the linear system for $v$. Note that although the linear system in (18) has a larger dimension than that of (17), it is sparse and can efficiently be solved by suitable direct [19] or iterative solvers [18].

$$
\left[\begin{array}{cc}
\alpha E_{1}-A_{1} & A_{2} \\
A_{3} & A_{4}
\end{array}\right]\left[\begin{array}{l}
v \\
\Lambda
\end{array}\right]=\left[\begin{array}{l}
B_{1} \\
B_{2}
\end{array}\right] b,
$$

Analogously, when we construct the left transformation matrix $T_{l}$ to obtain each term of (16), we can avoid of solving a liner system like $\left(\alpha E^{\mathrm{T}}-A^{\mathrm{T}}\right) w=C^{\mathrm{T}} c$. Instead, we solve the linear system for $w$. Once we have $T_{l}$ and $T_{r}$, then the reduce model (4) can be obtained by forming the reduce dimensional matrices as

$$
\left[\begin{array}{cc}
\alpha E_{1}^{T}-A_{1}^{T} & A_{3}^{T} \\
A_{2}^{T} & A_{4}^{T}
\end{array}\right]\left[\begin{array}{l}
w \\
\Lambda
\end{array}\right]=\left[\begin{array}{l}
C_{1}^{T} \\
C_{2}^{T}
\end{array}\right] c,
$$

$$
\begin{aligned}
& \hat{E}:=T_{l}^{T} E_{1} T_{r} \\
& \hat{A}:=\hat{A}_{1}-\hat{A}_{2} A_{4}^{-1} \hat{A}_{3}, \quad \hat{B}:=\hat{B}_{1}-\hat{A}_{2} A_{4}^{-1} \hat{B}_{2} \\
& \hat{C}:=\hat{C}_{1}-\hat{C}_{2} A_{4}^{-1} \hat{A}_{3}, \quad \hat{D}_{a}:=D_{a}-C_{2} A_{4}^{-1} B_{2},
\end{aligned}
$$

where $\hat{A}_{1}=T_{l}^{T} A_{1} T_{r}, \hat{A}_{2}=T_{l}^{T} A_{2}, \hat{A}_{3}=A_{3} T_{r} \quad$, and $\hat{B}_{1}=T_{l}^{T} B_{1}, \hat{C}_{1}=C_{1} T_{r}$.

The whole procedure to obtain the reduced ODE system (4), for a given index 1 descriptor system (1) is shown in Algorithm 2. However, a simple algebraic manipulation represents the ROM (4) as in the reduced index 1 DAE setting:

$$
\begin{aligned}
& {\left[\begin{array}{ll}
\hat{E} & 0 \\
0 & 0
\end{array}\right]\left[\begin{array}{l}
\dot{\hat{x}}_{1}(t) \\
\dot{\hat{x}}_{2}(t)
\end{array}\right]=\left[\begin{array}{cc}
\hat{A} & \hat{A}_{2} \\
\hat{A}_{3} & A_{4}
\end{array}\right]\left[\begin{array}{l}
\hat{x}_{1}(t) \\
\hat{x}_{2}(t)
\end{array}\right]+\left[\begin{array}{l}
\hat{B}_{1} \\
B_{2}
\end{array}\right] u(t),} \\
& \hat{y}(t)=\left[\begin{array}{ll}
C_{1} & C_{2}
\end{array}\right]\left[\begin{array}{l}
x_{1}(t) \\
x_{2}(t)
\end{array}\right] .
\end{aligned}
$$

\section{NUMERICAL RESUlTS}

\section{Algorithm 2: IRKA for index 1 DAE systems}

Input: $E_{1}, A_{1}, A_{2}, A_{3}, A_{4}, B_{1}, B_{2}, C_{1}, C_{2}$ from (1).

Output: Reduce dimensional matrices $\hat{E}, \hat{A}, \hat{B}, \hat{C}$ as in (20).

1. Select initial interpolation points $\sigma_{i} \stackrel{r}{i=1}$, and tangent directions $\quad b_{i}{ }_{i=1}^{r}$, and $c_{i}{ }_{i=1}^{r}$.

2. for $i=1,2, \ldots ., \mathrm{r}$ do

3. Solve the linear systems

$$
\begin{aligned}
& {\left[\begin{array}{cc}
\alpha E_{1}-A_{1} & A_{2} \\
A_{3} & A_{4}
\end{array}\right]\left[\begin{array}{l}
v_{i} \\
\Lambda
\end{array}\right]=\left[\begin{array}{l}
B_{1} \\
B_{2}
\end{array}\right] b, \text { for } v_{i}, \text { and }} \\
& {\left[\begin{array}{cc}
\alpha E_{1}^{T}-A_{1}^{T} & A_{3}^{T} \\
A_{2}^{T} & A_{4}^{T}
\end{array}\right]\left[\begin{array}{l}
w_{i} \\
\Lambda
\end{array}\right]=\left[\begin{array}{l}
C_{1}^{T} \\
C_{2}^{T}
\end{array}\right] c, \text { for } w_{i} .}
\end{aligned}
$$

4. Construct

$$
T_{r}=\left[v_{1}, v_{2}, \ldots . ., v_{r}\right], \quad T_{l}=\left[w_{1}, w_{2}, \ldots . ., w_{r}\right] .
$$

\section{5. end for}

6. while (not converge) do

7. Form $\hat{E}, \hat{A}, \hat{B}, \hat{C}$ as in (20).

8. Compute

$$
\hat{A} z_{i}=\hat{\lambda}_{i} \hat{E} z_{i}, \text { and } y^{*} \hat{A}=\hat{\lambda}_{i} y^{*} \hat{E} z_{i} .
$$

9. $\sigma_{i} \leftarrow-\hat{\lambda}_{i}, \quad b_{i}^{T} \leftarrow y^{*} \hat{B}$, and $c_{i} \leftarrow \hat{C} z_{i}$

10. Go to step 2

$$
\text { for } i=1,2, \ldots ., \mathrm{r} \text {. }
$$

11. $i=i+1$

12. end while

13. Form the reduced matrices as in (20).

To assess the performance of the techniques, this section discusses some numerical tests. The method is applied to a set 
of data introduced in [5]. The dimension of the original model is 7135, where the number of differential and algebraic variables are respectively, $n_{1}=606$ and $n_{2}=6529$. We compute exemplary reduce order model of dimension 50 , using Algorithm 2. The computations are carried out using MATLAB 7.11.0 (R2010b) on a board with 2 Intel Xeon X5650 CPUs with a 2.67-GHz clock speed.

Fig. 1 shows the frequency responses (largest singular value of $G(\omega)$ of full and 50 dimensional reduce order models in frequency domain over the frequency $(\omega)$ range of $10^{-2}$ to $10^{2}$.

In Fig. 2, the absolute error between the frequency responses of full and reduce models are shown. The relative error in the frequency responses of reduce dimensional model and full model are depicted in Fig. 3.

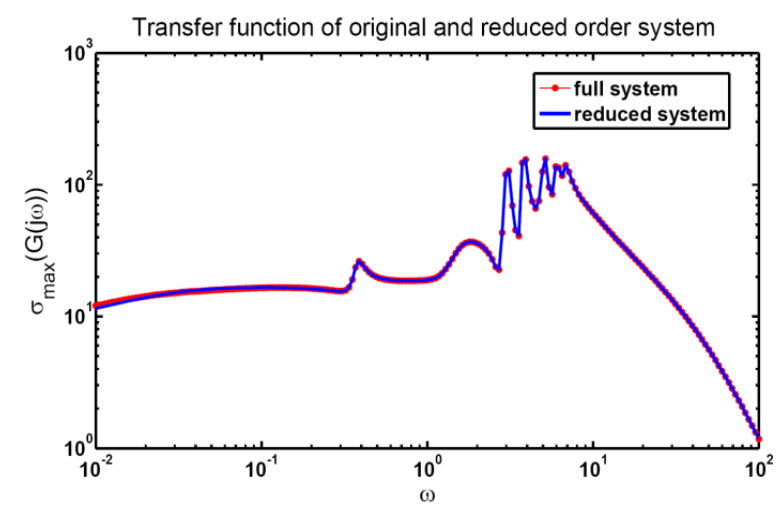

Fig. 1. Sigma plot (maximum singular values) of full and reduce order models.

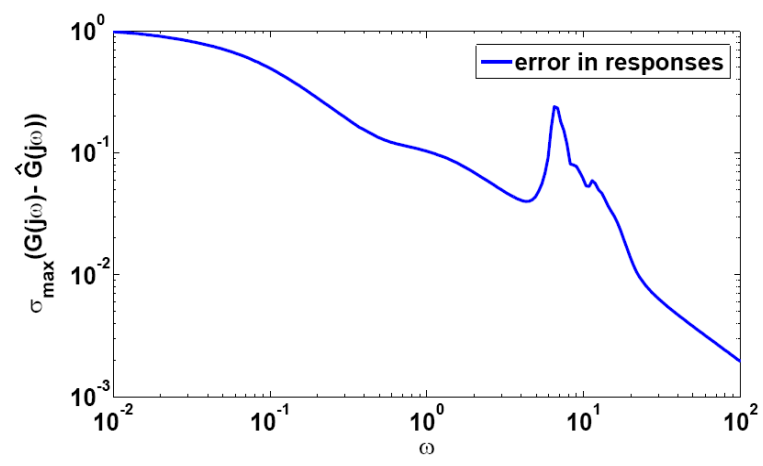

Fig. 2. Absolute error in the sigma plot of full and reduce order models.

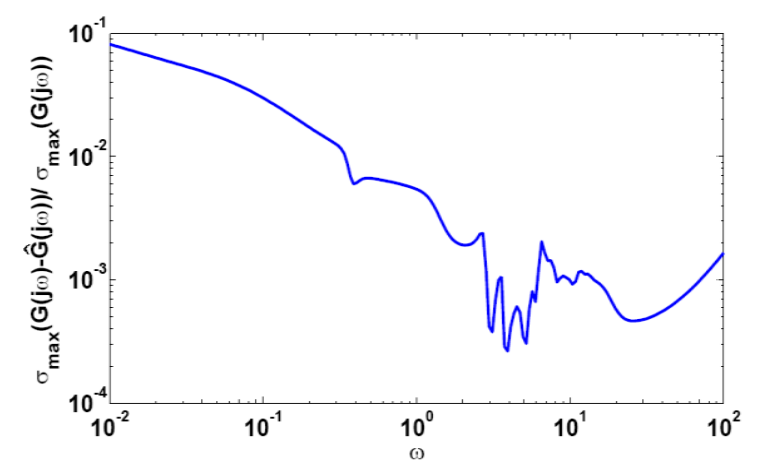

Fig. 3. Relative error in the sigma plot of full and reduced order models.

Since our model is a MIMO, we are also interested to analyze the one-to-one input-output behaviors of the full and reduce dimensional models. Considering the single input single output relations, for example, input 1 to output 2, Fig. 4 shows the time domain response of reduce dimensional model nicely matches that of the full model.

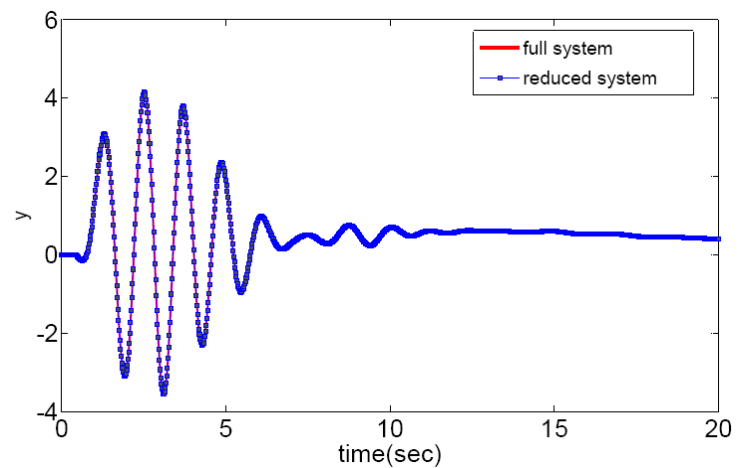

Fig. 4. Time domain response from input 1 to output 2 of full system and reduce system.

Fig. 5 and Fig. 6, respectively, show absolute and relative deviations between full and reduced models. Note that for time domain simulation we apply an implicit Euler method with fixed time step size $10^{-2}$.

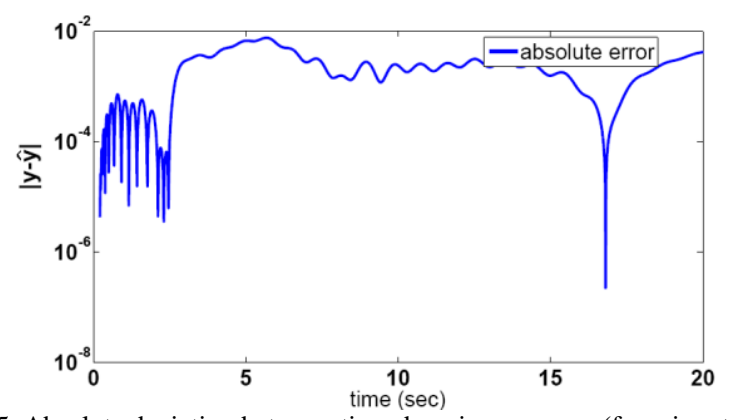

Fig. 5. Absolute deviation between time domain responses (from input 1 to output 2) for the full and reduce systems.

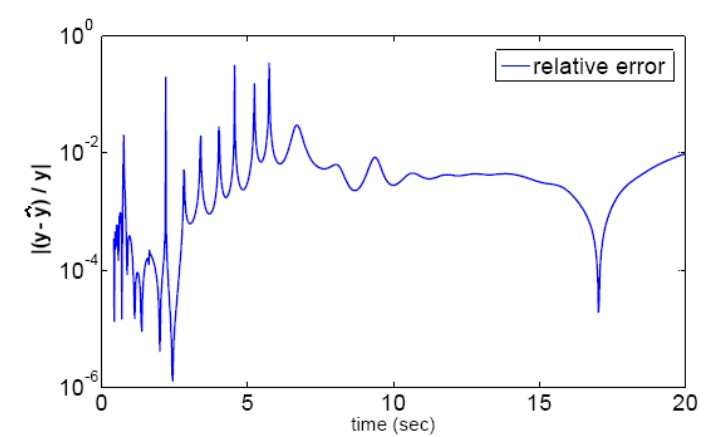

Fig. 6. Relative deviation between time domain responses (from input 1 to output 2) for the full and reduce systems.

\section{CONCLUSION}

We have presented an efficient reduction technique for An index 1 DAEs system, which in particular, arises from linearization of power system models around an equilibrium point [1].

A balancing based criterion for such models has been shown in [5]. There the main drawback is solving two continuous time algebraic Lyapunov equations. Based on existed techniques, here we have investigated interpolatory projection method via IRKA which is computationally cheap. Our strategy allows to work on the original matrices of the descriptor system and exploit their sparsity in the solver.

We have presented an algorithm to obtain reduced standard ODE systems. We also have shown how to extract the index 1 descriptor systems from the reduced ODE systems. The efficiency and accuracy has been demonstrated 
for one large model with 7135 degrees of freedom.

\section{ACKNOWLEDGMENT}

The authors express their thanks to Joost Rommes one of the authors in [5] for providing the models used in the numerical Experiments, and for valuable suggestion at different stages of this research work.

\section{REFERENCES}

[1] N. Martins, "Efficient eigenvalue and frequency response methods applied to power system small-signal stability studies," IEEE Transactions on Power Systems, vol. 1, no. 1, pp. 217-224, 2007.

[2] F. Freitas and A. Costa, "Computationally efficient optimal control methods applied to power systems," in Proc. IEEE 20th International Conference on Power Industry Computer Applications, 1997, pp. 287-294

[3] G. M. Huang, L. Zhao, and X. Song, “A new bifurcation analysis for power system dynamic voltage stability studies," in Proc. 2002 IEEE Power Engineering Society Winter Meeting, vol. 2, pp. 882-887, 2002.

[4] S. Costa, F. D. Freitas, and A. S. Silva, "Design of decentralized controllers for large power systems considering sparsity," IEEE Transactions on Power Systems, vol. 12, no. 1, pp. 144-152, February 1997.

[5] F. Freitas, J. Rommes, and N. Martins, "Gramian-based reduction method applied to large sparse power system descriptor models," IEEE Trans. Power Systems, vol. 23, no. 3, pp. 1258-1270, August 2008.

[6] M. M. Uddin, "Model reduction for Piezo-Mechanical system using balanced trancation," master's thesis, Stockholms University, Stockholm, Sweden, 2011.

[7] A. C. Antoulas, Approximation of Large-Scale Dynamical Systems. Philadelphia, PA: SIAM Publications, 2005, ch. 7, pp. 207-242.

[8] P. Benner, V. Mehrmann, and D. Sorensen, Dimension Reduction of Large-Scale Systems, Springer-Verlag, Berlin/Heidelberg, Germany, 2005, vol. 45, ch. 1-10, pp. 5-261.

[9] W. H. A. Schilders, H. A. V. D. Vorst, and J. Rommes, Model Order Reduction: Theory, Research Aspects and Applications. Berlin, Heidelberg: Springer-Verlag, 2008, part II and III, pp. 49-447.

[10] P. Benner, M.-S. Hossain, and T. Stykel, "Model reduction of periodic descriptor systems using balanced truncation, Model Reduction in Circuit Simulation, 2011, vol. 74, ch. 11, pp. 193-206.

[11] D. C. Villemagne and R. E. Skelton, "Model reduction using a projection formulation," Internat. J. Control, vol. 46, pp. 2141-2169, 1987.

[12] E. J. Grimme, "Krylov projection methods for model reduction," $\mathrm{PhD}$ Thesis, Univ. of Illinois at Urbana-Champaign, USA, 1997.

[13] A. Ruhe, "Rational Krylov algorithms for nonsymmetric eigenvalue problems II: Matrix pairs," Linear Algebra Appl., vol. 197, pp. 283-295, 1994.
[14] P. Feldmann and R. W. Freund, "Efficient linear circuit analysis by Pad'eapproximation via the Lanczos process," IEEE Trans. Comput.-Aided Des. Integr. Circuits Syst., vol. 14, no. 5, pp. 639-649, 1995.

[15] S. Gugercin, A. C. Antoulas, and C. Beattie, "H2 model reduction for large-scale dynamical systems," SIAM J. Matrix Anal. Appl., vol. 30, no. 2, pp. 609-638, 2008

[16] K. Gallivan, A. Vandendorpe, and P. V. Dooren, "Model reduction of MIMO systems via tangential interpolation," SIAM J. Matrix Anal. Appl., vol. 26, no. 2, pp. 328-349, 2004.

[17] S. Gugercin, T. Stykel, and S. Wyatt, "Model reduction of descriptor systems by interpolatory projection methods," SIAM J. Sci. Comput., vol. 35, no. 5, pp. B1010-B1033, 2013.

[18] Y. Saad, Iterative Methods for Sparse Linear Systems. Philadelphia, PA, USA: SIAM, 2003, ch. 4-7, pp. 95-227.

[19] T. A. Davis, Direct Methods for Sparse Linear Systems (Fundamentals of Algorithms 2). Philadelphia, PA, USA: SIAM, 2006, ch. 8, pp. 135-144.

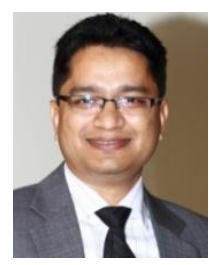

Mohammad Sahadet Hossain was born in Munshigonj, Dhaka, Bangladesh in 1978. He worked as a postdoc research scientist at the International Max-Planck Institute for Dynamics of Complex Technical Systems, Magdeburg, Germany in 2011 to 2012. Before that he obtained his PhD from Chemnitz University of Technology, Germany in 2011. Before that he obtained his $\mathrm{PhD}$ from Chemnitz University of Technology, Germany in 2011. Dr. Hossain is presently working as an assistant professor at the Department of Mathematics and Physics, North South University, Dhaka, Bagladesh. His research interest includes control theory and engineering, reduced order modeling of periodic systems, iterative based solution of large sparse periodic matrix equation, and development of numerical algorithms for reduced order modeling of time-varying dynamical systems.

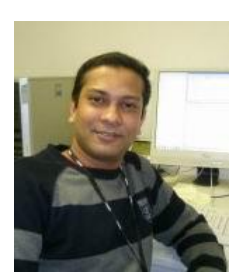

M. Monir Uddin was born in Sandwip, Chittagong, Bangladesh in 1978. He is a $\mathrm{PhD}$ candidate in the research group of Computational Methods in Systems and Control Theory, at the Max Planck Institute for Dynamics of Complex Technical Systems, Magdeburg, Germany.

Before that he received his master of science in applied mathematics from Stockholm University, Stockholm, Sweden in 2010. Mr. Uddin is also an associate member of International Max Plank Research School (IMPRS) Magdeburg, Germany. His fields of research interests include systems and control theory with application in model order reduction, numerical linear algebra, optimization, and scientific computing involves in iterative solutions of large matrix equations. 